Para enlazar con este artículo / To link to this article:

http://dx.doi.org/10.14198/fem.2018.31.11

Para citar este artículo / To cite this article:

Torres González, Obdulia. En Feminismo/s, 31 (junio 2018): 231-250, DOI: 10.14198/fem.2018.31.11

\title{
LA SEGREGACIÓN HORIZONTAL: EL RIESGO DE LOS AGREGADOS ESTADÍSTICOS ${ }^{1}$
}

\author{
HORIZONTAL SEGREGATION STUDIES: \\ THE RISK OF USING STATISTICAL AGGREGATES
}

\author{
Obdulia TORRES GONZÁLEZ \\ Universidad de Salamanca \\ https://orcid.org/0000-0003-1620-6911
}

\section{Resumen}

En este artículo se muestra el riesgo de usar agregados estadísticos en los estudios de segregación horizontal. El análisis sugiere que su uso puede llevar a conclusiones erróneas y a medidas políticas inadecuadas. La segregación horizontal es más que la infrarrepresentación de la mujer en los campos STEM, también afecta a algunos campos de las Humanidades y las Ciencias Sociales. Se considera, además, que el énfasis prestado a los estudios STEM ha influido en la dirección que ha tomado la agenda de investigación: el factor fundamental estudiado es el desempeño en Matemáticas por parte de las mujeres y éste no explica su diferente participación en Humanidades o Ciencias Sociales. En los estudios de segregación horizontal se debe prestar igual atención a las elecciones de chicos y chicas. Finalmente, se analiza brevemente los fenómenos de segregación vertical en campos feminizados y masculinizados.

Palabras clave: Segregación horizontal, elección vocacional chicos, desempeño en Matemáticas, campos masculinizados, análisis estadístico.

1. Este artículo se ha realizado en el marco del Proyecto de I+D "La frontera entre ciencia y política y la ciencia en la frontera: la ciencia española, 1907-1975”, FFI2015-64529-P (MINECO/FEDER)

Los contenidos de la revista se publican bajo una licencia de Creative Commons Reconocimiento 4.0 Internacional (CC BY 4.0)

Feminismo/s 31, junio 2018, pp. 231-250 


\begin{abstract}
This article shows the risk of using statistical aggregates in horizontal segregation studies. The analysis suggests that its use can lead to erroneous conclusions and inappropriate policy measures. Horizontal segregation is more than the underrepresentation of women in the STEM fields, it also affects some fields of the humanities and social sciences. It is also considered that the emphasis given to STEM studies has influenced the direction taken by the research agenda: the fundamental factor studied is the performance in mathematics by women and this does not explain the different participation of women in humanities or social sciences. In studies of horizontal segregation, equal attention must be paid to the choices of boys and girls. Finally, the phenomena of vertical segregation in feminized and masculinized fields are briefly analyzed.
\end{abstract}

Keywords: Horizontal segregation, boys' career choices, mathematical performance, masculinized fields, statistical analysis.

\title{
1. INTRODUCCIÓN
}

Los análisis de segregación horizontal se han centrado casi exclusivamente en las disciplinas STEM ${ }^{2}$, es decir, en la escasa presencia de mujeres en Ciencia e Ingeniería, y pasan por alto que en algunas humanidades la presencia femenina es equiparable a la que tienen cualquiera de estos campos, o que en algunas ingenierías, como la Arquitectura, su presencia asciende a un 50\%, o que el porcentaje de estudiantes mujeres en Matemáticas es del 38\%.

Los responsables de esta situación son los agregados estadísticos que usamos como base de análisis y que provienen de los manuales para la medición de las actividades de investigación y desarrollo, fundamentalmente el manual de Frascati y el de Camberra.

Por ejemplo, el último informe de la OCDE para España afirma:

En 2015, las mujeres están infrarrepresentadas en el campo de estudio STEM, sobre todo en Tecnologías de la información y la comunicación (TIC), ya que solo el 12\% de los matriculados por primera vez eran mujeres, y en el de Ingeniería, industria y construcción con un $24 \%$ de mujeres. Sin embargo, las mujeres están sobrerrepresentadas en los campos de la Educación (79\%) y en el de la Salud y bienestar (72\%). (MECD 22)Nos hemos acostumbrado

2. Ciencia, tecnología, ingeniería y matemáticas por sus siglas en inglés.

Feminismo/s 31, junio 2018, pp. 231-250 
a ver estos agregados como un todo homogéneo cuando lo cierto es que esconden una enorme variabilidad en su seno en lo que se refiere a la participación de las mujeres.

En este artículo se muestran las diferencias estadísticas en los distintos subcampos dentro de los agregados estadísticos, haciendo hincapié en que la segregación horizontal es más que la infrarrepresentación de la mujer en los campos STEM, y que también afecta a las Humanidades y a las Ciencias Sociales. En segundo lugar, se considera que el énfasis prestado a los estudios STEM ha influido en la dirección que ha tomado la agenda de investigación de los factores explicativos de la discriminación horizontal. Esta investigación se ha centrado prácticamente en el desempeño en Matemáticas por parte de las mujeres, factor que no explica el resto de las diferencias que encontramos en los agregados y que ha favorecido la presencia de nuevos estereotipos de género. En tercer lugar, se considera que en los estudios de segregación horizontal se debe prestar igual atención a las elecciones de chicos y chicas. Muchos campos considerados tradicionalmente masculinos se han feminizado, pero pocos campos en los que han predominado las mujeres son demandados por los hombres. El análisis de este tipo de fenómenos ayudaría a comprender mejor los fenómenos de segregación. Finalmente, se analiza brevemente los fenómenos de segregación vertical en campos feminizados y masculinizados.

Los agregados estadísticos son ciertamente útiles, sin su ayuda contaríamos con una cantidad de variables prácticamente inmanejables, pero en el tema que nos ocupa no parece que estén siendo de utilidad. El análisis de la evolución de los datos de segregación horizontal muestra que la situación prácticamente no ha variado desde finales de los años 90. Lo que quiere decir que los programas y medidas propuestas no están funcionando. Un análisis más detallado de la estadística puede propiciar un cambio en la agenda de investigación y en la agenda política en cuestiones de ciencia y género.

\section{METODOLOGÍA}

Los datos para el estudio estadístico están tomados de las bases de datos del Instituto Nacional de Estadística (INE) y del Ministerio de Educación (MECD). En el caso del profesorado, refieren al personal docente e investigador de los 
centros propios de las universidades públicas, quedan por tanto excluidos el personal de los centros adscritos de las universidades públicas y todo el personal perteneciente a las universidades privadas, dado que no existe un recuento del personal total desagregado por género. En el caso del alumnado se toma el número de estudiantes matriculados en grados por ámbito de estudio, que remite a: Arte y Humanidades, Educación, Ciencias Sociales y Jurídicas, Ciencias, Ingeniería, Industria y Construcción, Agricultura y Veterinaria, Salud y Servicios Sociales, y Servicios, según la clasificación de la UNESCO. En ocasiones se ha procedido a agruparlos manualmente para que coincidan con las 5 ramas de enseñanza en las que se agrupa al personal docente, según el RD 415/2015, en un curso académico determinado.

Salvo que se indique lo contrario, todos los datos pertenecen al curso 2015-2016.

Cuando hablamos de rama de conocimiento, el cálculo de los totales puede ser ligeramente distinto de los cálculos que se obtienen a través de la página del ministerio y del INE. El objetivo de este trabajo es el estudio de los agregados estadísticos y el análisis del peso que las distintas áreas de conocimiento tienen en ese agregado, por lo que se ha procedido a agrupar las áreas de conocimiento por rama manualmente según el Real Decreto antes citado, dado que ese nivel de desagregación no se ofrece en la página del Ministerio. Esa descomposición puede generar ligeras diferencias con los datos oficiales.

\section{LOS DATOS DE LA SEGREGACIÓN HORIZONTAL}

La segregación horizontal se refiere a la concentración de hombres o mujeres en campos específicos, relacionados con estereotipos sexuales, que se basan en supuestas preferencias o habilidades en su desempeño. Así, los hombres eligen ingenierías en razón de su mejor capacidad espacial y matemática, o las mujeres eligen educación porque prefieren el trato con los niños. Pero cuando hablamos de estadísticas, raramente nos referimos a disciplinas concretas, dado que el volumen de datos sería muy difícil de manejar. La UNESCO, a través de la clasificación internacional normalizada de educación, agrupa los diferentes campos educativos en 8 grupos: (1) Educación, (2) Arte y Humanidades, (3) Ciencias Sociales, Educación comercial y Derecho, (4) Ciencias, (5) Ingeniería, Industria y construcción, (6) Agricultura, (7) Salud 
y servicios sociales y (8) Servicios. Estos 8 sectores se reducen a 6 cuando pasamos a la medición de los recursos humanos en los sistemas de I+D tal como los regula el manual de Camberra (OCDE). Aquí educación se incluye en ciencias sociales y el sector servicios desaparece. Este es el nivel de agregación usado por la OCDE y Eurostat tal como lo recomienda además la nueva versión del manual de Frascati (OCDE). Cada uno de estos grupos tiene a su vez dos niveles de desagregación, por subcampos y por materias ${ }^{3}$, pero como se señaló, la estadística se refiere a los grandes campos de agregación. Implícitamente se asume que hay una cierta uniformidad en estos campos en lo que a elección vocacional se refiere. A continuación, se muestra una evolución de los indicadores de discriminación horizontal.

En el curso 1982-83, (...) El porcentaje de mujeres en cada área era el siguiente: el 47,0\% de los estudiantes de ciencias experimentales eran mujeres, en ciencias de la salud son el 49,9\%, el 11,1\% en Escuelas Técnicas Superiores (E.T.S.), el 44,5\% en ciencias jurídicas y sociales y el $63,5 \%$ en Humanidades. (...) En el curso 1990-91, las mujeres son ya mayoría en la universidad española (...) Esa mayoría es patente en todas las áreas, excepto en las E.T.S., donde sólo son un 20,6\% y en las ciencias experimentales, donde son un 48,1\%; en ciencias de la salud constituyen el $64,7 \%$, en ciencias jurídicas y sociales el 59,3\% y en humanidades el 64,5\%. (Pérez Sedeño)

Estos son prácticamente los primeros datos con los que se cuenta de segregación horizontal en España. No están disponibles en la web del Instituto Nacional de Estadística, las autoras los extrajeron de las memorias de los años correspondientes (editadas en papel) que están en el centro de investigación y

3. A modo de ejemplo, estos serían los niveles de desagregación del campo de ciencias: 42 Ciencias de la Vida: Biología, Botánica, Bacteriología, Toxicología, Microbiología, Zoología, Entomología, Ornitología, Genética, Bioquímica, Biofísica, otras ciencias afines, excepto Medicina y Veterinaria. 44 Ciencias Físicas: Astronomía y Ciencias Espaciales, Física y asignaturas afines, Química y asignaturas afines, Geología, Geofísica, Mineralogía, Antropología Física, Geografía Física y demás Ciencias de la Tierra, Meteorología y demás Ciencias de la Atmósfera, comprendida la investigación sobre el clima, las Ciencias Marinas, Vulcanología, Paleoecología. 46 Matemáticas y Estadística: Matemáticas, Investigación de Operaciones, Análisis Numérico, Ciencias Actuariales, Estadística y otros sectores afines. 48 Informática: Concepción de Sistemas, Programación Informática, Procesamiento de Datos, Redes, Sistemas Operativos - elaboración de programas informáticos solamente (el material y equipo se deben clasificar en el sector de la Ingeniería).

Feminismo/s 31, junio 2018, pp. 231-250 
documentación educativa (CIDE) del MECD ${ }^{4}$. A continuación, se muestra la evolución de la segregación horizontal desde que contamos con datos online desagregados por género.

\begin{tabular}{|l|c|c|}
\cline { 2 - 3 } \multicolumn{1}{c|}{} & \multicolumn{2}{c|}{ Curso académico } \\
\hline Rama de conocimiento & $1998-99^{5}$ & $2015-16$ \\
\hline Ciencias & 48,8 & 37,1 \\
\hline Arte y Humanidades & 64,9 & 61,4 \\
\hline Ciencias Sociales & 59,8 & 61,4 \\
\hline Ingeniería & 29,5 & 28,9 \\
\hline Agricultura & 39,8 & 47,3 \\
\hline Ciencias salud & 74,8 & 72,8 \\
\hline
\end{tabular}

Tabla 1. Evolución del porcentaje de mujeres por rama de conocimiento.

Un rápido vistazo a los datos indica que la situación no parece haber sufrido cambios sustanciales, de hecho, el porcentaje de mujeres en ciencias empeora, dado que cae 10 puntos respecto a los datos de los años 80 . Se incrementa en el resto de las áreas (excepto en Humanidades, lo que es fácilmente explicable dada la disminución de oportunidades en el mercado laboral para sus egresados) y llega a ser mayoría excepto en Ingeniería. A finales de la década de los 90 se ha consolidado la feminización de Ciencias de la Salud, situación que pervive hasta la fecha. En el resto de los campos, en los 16 años transcurridos no hay cambios significativos, las variaciones porcentuales son mínimas y no se aprecian tendencias de cambio.

Ya desde el Informe ETAN (European Commision, 1999), donde se inicia la definición de indicadores para las cuestiones de ciencia y género, se propone un indicador de segregación horizontal al que denominan índice de disimilitud. La forma de medir esta segregación es calcular la tasa de

4. Comunicación personal.

5. Hemos de tener en cuenta que en el curso 98-99 hablamos de estudios de licenciatura y diplomatura. Se han unido ambos por rama. La composición de las ramas también es ligeramente diferente: para afinar la comparabilidad con el sistema actual Ingeniería Informática, se ha añadido a la rama de Ciencias; igualmente, Ingeniería Agrónoma se elimina de Ingeniería y se pasa a Agricultura. 
concentración de mujeres en los distintos sectores o disciplinas. Según dicho informe, el índice de disimilitud "proporciona una medida teórica del porcentaje de mujeres y hombres en un campo dado, que tendría que moverse a otro campo de la ciencia para asegurar que la proporción de mujeres fuera la misma en todos los campos." (European Commision, Science Policy 57) A mayor valor del índice mayor disimilitud, dado que multiplicado por 100 nos da el porcentaje de personas que tienen que moverse de un campo a otro para conseguir una proporción igual en todos los campos. El índice se calcula hallando el porcentaje de mujeres en cada campo respecto al porcentaje total de mujeres en el sector estudiado y hallando la diferencia respecto al mismo cálculo realizado para los varones. Los valores absolutos de esas diferencias se suman y el resultado se divide por dos. El hecho de trabajar con valores absolutos significa que todas las diferencias se suman y no se compensan valores de un campo científico a otro ${ }^{6}$. El índice de disimilitud para el curso 98-98 era de 0.15 . Ello significa que un $15 \%$ de alumnos y alumnas deberían cambiar de carrera para que haya un $57.5 \%$ de estudiantes mujeres en cada campo ${ }^{7}$. Por su parte, el índice de disimilitud para el curso 15-16 es de 0.25, lo que implicaría que un $25 \%$ de estudiantes debería cambiar de campo para que hubiera un 54\% de mujeres en cada campo. Según este indicador la segregación horizontal ha aumentado en España en los últimos 15 años.

La ley 3/2007 para la igualdad efectiva de mujeres y hombres considera: "A los efectos de esta Ley, se entenderá por composición equilibrada la presencia de mujeres y hombres de forma que, en el conjunto a que se refiera, las personas de cada sexo no superen el sesenta por ciento ni sean menos del cuarenta por ciento". Atendiendo a esto, y salvo en las ramas de Ciencias de la Salud e Ingeniería, parece que hemos alcanzado una composición equilibrada de los distintos sectores de estudio. A continuación, analizaremos algunos de estos agregados para saber cuáles son las elecciones de los estudiantes y si realmente hemos acabado con el sesgo de género en la elección de estudios.

La rama de Arte y Humanidades presenta un promedio de estudiantes matriculadas del $61 \%$. Resulta sorprendente observar que el porcentaje de

6. Para una revisión crítica de este indicador ver Torres.

7. Para este curso se suma licenciatura y diplomatura, lo que arroja un 57,5\% de estudiantes mujeres en las aulas.

Feminismo/s 31, junio 2018, pp. 231-250 
mujeres en las aulas de Filosofía es de un 36\%, aunque un poco peor es la situación en Historia, donde constituyen el 33\% del alumnado en sus aulas.

Ciencias Sociales presenta igualmente un promedio de $61 \%$, pero el porcentaje en Educación es de un 78\% de hecho, si eliminamos Educación del cómputo el promedio cae 5 puntos. Es curioso observar como la proporción de hombres que elige Educación va cayendo a medida que retrocedemos en las etapas educativas llegando a menos de un 7\% en Educación Infantil. Lo mismo ocurre con Psicología, donde los varones solo son el 27\% del alumnado matriculado.

El 37\% de estudiantes mujeres en Ciencias se debe fundamentalmente al peso que tiene Informática en el cómputo (12\% de mujeres matriculadas). Si eliminamos Informática del cálculo subimos a un $49 \%$. Otra disciplina muy alejada del promedio, pero en sentido contrario es Biología, que cuenta con un $62 \%$ de alumnado femenino, y que cuando se elimina transforma ese $37 \%$ en un 33\%. Debemos hacer hincapié en los 36 puntos porcentuales de diferencia que existen entre Biología y Física, dos campos que pertenecen al mismo agregado estadístico.

Dentro de Ciencias de la Salud tenemos un 66\% en Medicina, llegamos al 73\% cuando incluimos Enfermería (80\% de mujeres) o Farmacia (70\%).

La situación no es homogénea ni siquiera en las ingenierías, los auténticos feudos masculinos parecen estar en Electricidad y Energía, y en Electrónica y Automática, con menos de un 18\% de mujeres. Por el contrario, en Arquitectura hay un 50\% de estudiantes mujeres y en Industria de la Alimentación un 66\%.

Como se puede observar, hay diferencias significativas entre los campos pertenecientes a todos y cada uno de los agregados y además hay estudios que tienen un enorme peso en el promedio final (Informática, Biología o Enfermería, por ejemplo). No contemplar estas diferencias implica que las medidas políticas que se tomen para acabar con la segregación horizontal no serán efectivas, porque estamos tratando como uniformes campos que distan mucho de serlo. Debemos pasar de la pregunta ¿por qué hay tan pocas mujeres en Ingeniería o en Ciencias? a cuestiones más concretas como ¿por qué Ciencias Químicas tiene un 53\% de estudiantes mujeres y Física un 25\%?, ¿por qué las mujeres son un $62 \%$ en los estudios de Biología? o ¿por qué hay tan pocas estudiantes de Filosofía?

Feminismo/s 31, junio 2018, pp. 231-250 
¿A qué obedecen tales disparidades en campos que se suponían homogéneos? ¿Qué subyace a la elección de carrera de chicos y chicas?

\section{EL DESEMPEÑO EN MATEMÁTICAS COMO FACTOR EXPLICATIVO}

La preocupación exclusiva por los campos STEM como indicativos de las desigualdades de género en los sistemas de investigación, ha hecho que la investigación acerca de los factores explicativos de tales desigualdades se centre en estos campos. Entre los factores que se han señalado destacan las diferencias de género en habilidades cognitivas. La cuestión es si existen esas diferencias y si las mismas explican la inferior presencia de las mujeres en las carreras de ciencias.

Las explicaciones tradicionales que intentan dar cuenta de las desigualdades de género en los distintos estudios se han centrado en las diferencias en habilidades cognitivas, herederas de los tests psicométricos de principios de siglo XX y de su fundamentación biologicista en la década de los 70:

[...] era la creencia en que existían diferencias en las capacidades y habilidades cognitivas de los géneros lo que se trataba de explicar a partir del supuesto de una lateralización cerebral distinta; y ello llevó a indagar en el contexto de las hormonas sexuales en busca de las causas de tal lateralización. (Gómez Rodríguez 128)

Lo que estas teorías afirman es que, debido a la lateralización cerebral fundamentada en la acción de las hormonas en el cerebro, los chicos son mejores en habilidades matemáticas y espaciales y las chicas en habilidades lingüísticas. Como señala la autora, esta teoría venía a fundamentar viejas asunciones acerca de los roles sociales y las elecciones de los distintos géneros, justificando bajo una cobertura científica lo que la mayoría de las veces eran simples procesos de discriminación. Los estudios de las diferencias en habilidades cognitivas resurgen más o menos periódicamente como el Fénix de sus cenizas ${ }^{8}$,

8. De hecho, el debate ha vuelto a reavivarse a raíz de la decisión de los NIH (National Institute of Health) americanos de exigir que se incluyan animales de ambos sexos en la investigación básica y en los ensayos preclínicos para obtener fondos de esa institución. Aunque era una demanda de muchas investigadoras y del movimiento feminista, dado el efecto desigual que algunas medicinas tienen en función del sexo del paciente, la medida provoca la desconfianza de algunos sectores ante el miedo de que una vez más

Feminismo/s 31, junio 2018, pp. 231-250 
pese a que la mayoría de los meta-análisis de la bibliografía lo descarta (NAS, European Commission 2012). Lo que está en juego aquí no es tanto la diferencia en habilidades cognitivas, sino en qué medida esas diferencias, estimadas a través de los tests psicométricos de inteligencia realizados en el bachillerato, sirven como predictores de las elecciones vocacionales que harán chicos y chicas al llegar a la educación terciaria. El supuesto que subyace es que el bajo rendimiento de las estudiantes en matemáticas, debido precisamente a diferencias cognitivas con los varones y explicadas fundamentalmente a través de diferencias estructurales cerebrales, explica su escasa presencia en las carreras de ciencia e ingeniería. Pero como señalan los últimos estudios, en la actualidad no hay diferencias en éxito académico en los aspectos señalados a nivel de bachillerato. De acuerdo con el informe de la Comisión Europea (basado en los últimos informes PISA), en algunos países de la UE las chicas puntúan igual o superior a sus compañeros varones. (European Commision, Meta-analisys of gender 73) lo que parece indicar que las diferencias se deben más a sistemas educativos que diferencias innatas por género.

En segundo lugar, no se ha probado que las medidas de aptitud sean un buen predictor de las elecciones de carreras de ciencia e ingeniería. En Estados Unidos no son los que obtienen puntuaciones más altas en el SAT-M ${ }^{9}$ los que después eligen ciencia e ingeniería. La estadística señala que menos de un tercio de los hombres que se dedican a ciencia e ingeniería obtuvo una puntuación por encima de los 650 puntos, que se considera el umbral mínimo para tener éxito en estos campos. Finalmente, en lo que los análisis sobre segregación horizontal parecen coincidir es en que los factores determinantes en la elección de carrera parecen estar más en las presiones e influencias sociales que reciben chicos y chicas y en los estereotipos negativos con los que las chicas se ven confrontadas, que en cualquier supuesta diferencia en habilidades cognitivas. (NAS 25-26)

las diferencias acaben siendo transformadas en desigualdades. El debate se puede seguir en la página web de OSSD (Organization for the Study of Sex Difference).

9. Viene a ser el equivalente estadounidense de nuestras pruebas de acceso a la universidad (Scholar Aptitude Test - Mathematics). 
Pero más allá de estos estudios, el peor desempeño de las mujeres en habilidades matemáticas y espaciales se ha establecido como un sólido estereotipo sexual y se ha constituido en una profecía autocumplida. Si atendemos a los datos, en Ciencias, Matemáticas y Computación, la Europa de los 15 arrojaba un promedio de 36\% en el año 2001 para estudiantes egresados de las antiguas licenciaturas (nivel ISCED 6) (European Commision, She 43). De hecho, para el curso 98-99 España contaba con un 52\% de mujeres estudiando Matemáticas. La figura 1 muestra la evolución de las estudiantes matriculadas en Matemáticas en España.

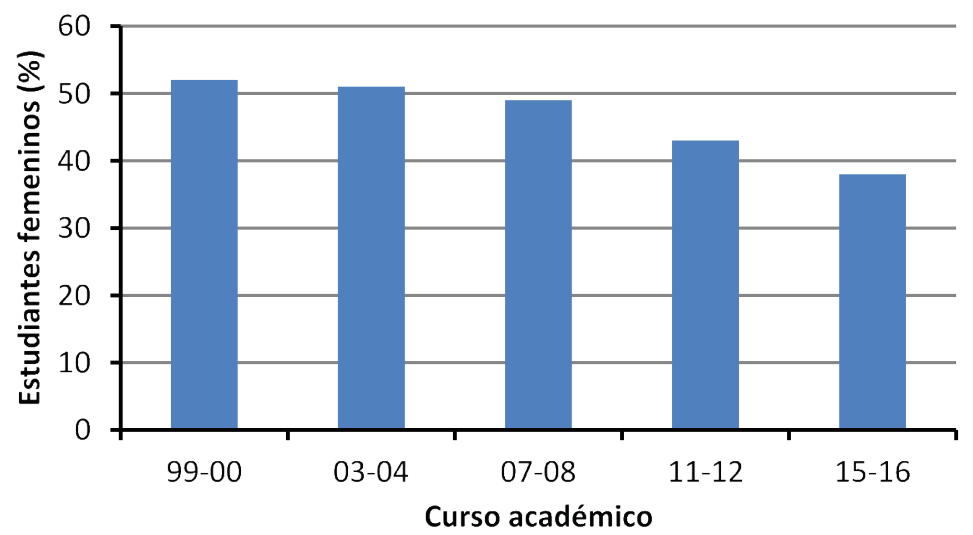

Figure 1. Evolución del porcentaje de mujeres matriculadas en matemáticas

Como se ve ha habido un descenso sostenido de las mismas. Parece que más que destruirse un estereotipo se ha creado uno, basado en la creencia en un desempeño inferior en Matemáticas, que parece estar desanimando a las estudiantes a iniciar esa carrera, dado que desde el año 98 se han perdido 14 puntos porcentuales en matrícula femenina ${ }^{10}$.

10. Los medios de comunicación alientan ese estereotipo cuando publican datos escasamente contrastados. Hace unos días El País digital afirmaba en uno de sus artículos: "Las matrículas universitarias de mujeres no superan el $30 \%$ en ninguna carrera de ciencia, tecnología, ingeniería y matemáticas" (Rubio) Lo que atendiendo a los datos que hemos desgranado más arriba no es cierto. Informaciones de este tipo refuerzan los estereotipos de las diferencias en habilidades cognitivas y desaniman a las 
Resulta asimismo sorprendente que se pretenda que los tests sean un indicador de la elección de carrera científica cuando se refiere a Ciencia e Ingeniería, pero se desprecie su efecto en la elección que hacen las estudiantes de Ciencias de la Salud. Si bien en el bachillerato de Ciencias Sociales los estudiantes tienen un temario de matemáticas diferentes a otras ramas, el bachillerato de Ciencias de la Salud y de Ciencias tiene Matemáticas como asignatura común y es bien sabido que Medicina acapara prácticamente los mejores expedientes del país con una nota de corte para acceder a la universidad superior a los 12 puntos $^{11}$.

El interés en el supuesto desigual desempeño en matemáticas de chicos y chicas ha tenido otra consecuencia importante, y es que ha dirigido la construcción de indicadores y los estudios de segregación horizontal. La construcción de indicadores se realiza desde un conjunto de asunciones acerca de una problemática en cuestión y de las posibles causas de la misma. Que las causas de la escasa presencia de la mujer en la ciencia se atribuyeran a un peor desempeño en Matemáticas ha tenido como consecuencia que no se exploren los agregados construidos más allá de los de Ciencias. De hecho, los únicos campos que se desagregan ya desde el segundo informe de She Figure en el 2006 son los de Ciencia e Ingeniería, que presenta datos desagregados por disciplinas: Ciencias Biológicas, Ciencias Físicas, Matemáticas y Estadística mientras que Ingeniería se descompone en Arquitectura y Construcción, Manufacturas y Procesamiento e Ingeniería. Las matemáticas son comunes a Ciencia e Ingeniería mientras que las habilidades lingüísticas son fundamentales en los estudios de Humanidades y Ciencias Sociales. Si no indagamos más allá de los agregados estadísticos construidos, tenemos una explicación de las elecciones vocacionales de hombres y mujeres, pero cuando descomponemos esos agregados, vemos que la razón aducida no explica por qué hay menos mujeres en Filosofía o Historia, porque hay un 50\% de mujeres arquitectas o un $60 \%$ de biólogas.

mujeres a emprender las carreras científicas. https://elpais.com/elpais/2017/11/22/ ciencia/1511369074_820675.html Último acceso 05/12/2017

11. Oscila entre un 12,1 para la Universidad de LLeida a un 12,8 de la Universidad de Valencia, para el curso 2016-2017. Fuente http://notasdecorte.es/medicina

Feminismo/s 31, junio 2018, pp. 231-250 
Realmente la infrarrepresentación de la mujer en la ciencia, atendiendo a los datos de España, se concentra en Física (26\%) e Informática (12\%). Si la segregación horizontal es definida como la elección sesgada de carrera por motivos de género, parece que tenemos que dar cuenta de la falta de mujeres en Física, Ingeniería e Informática, pero también en Filosofía o Historia. Tenemos que explicar por qué los chicos no eligen Educación o Psicología o por qué un $62 \%$ de mujeres elige Biología, ninguna de estas elecciones es explicada por el desempeño en Matemáticas. Pero lo más importante, en el análisis de la segregación horizontal deber ser analizadas tanto las elecciones femeninas como las masculinas y las razones que subyacen a ellas.

\section{LA ELECCIÓN DE ELLOS Y ELLAS}

Atendiendo a los datos de curso 2015-16, el porcentaje de mujeres que elige Ciencias de la Salud, sobre el total de mujeres en la universidad, es más del doble que el de hombres ( $16 \%$ frente a $7 \%$ ), mientras que el porcentaje de chicos que eligen Ingeniería casi triplica al de las chicas (25\% frente a 9\%). En la elección de Ciencias hay una diferencia de 1,5\% de diferencia a favor de ellos, pero en general ésta no es una opción mayoritariamente elegida por ninguno de los dos grupos (sólo un 8\% de los chicos). La opción mayoritaria para ambos grupos es Ciencias Sociales que es elegida por un $37 \%$ de varones y un $50 \%$ de las mujeres. Cuando descomponemos este campo, vemos que la misma proporción de chicos y chicas (22\%) eligen Administración, Empresa y Derecho. La diferencia entre ambos en lo que respecta al agregado estriba nuevamente en el peso que tiene Educación y Psicología.

Mucho se ha escrito acerca de los factores que explican las elecciones de las chicas, estereotipos de género, expectativas parentales, sesgo de los profesores en la enseñanza secundaria, presión de los padres, influencia de los medios de comunicación, necesidad de aceptación social, pero muy poco se ha investigado acerca de por qué los chicos no eligen profesiones feminizadas como Educación, Psicología o Enfermería. De hecho la revisión que se realiza a cargo de la Unión Europea de la investigación realizada en cuestiones de género no recoge ningún estudio posterior a 2003 y la mayoría data de finales de los 80 y principios de los 90 .

Hayes realiza una extensa revisión donde se incluyen las razones que mueven a los hombres a no elegir profesiones feminizadas. Las dos principales

Feminismo/s 31, junio 2018, pp. 231-250 
razones aducidas son la pérdida de ingresos y por tanto de estatus, y los estereotipos de género, por los que el hombre es el encargado de llevar el dinero a casa. Puede pensarse que la literatura revisada por la autora se encuentra obsoleta, desgraciadamente las razones aducidas parecen ser completamente válidas en 2017: brecha salarial (según datos de Eurostat para el 2015 las mujeres cobran alrededor de un 15\% menos que los hombres) y estereotipos de género. Según el último Eurobarómetro especial "Gender Equality 2017. Gender Equality, Stereotypes, and Women in Politics", el 44\% de los europeos piensa que el papel fundamental de la mujer es cuidar de la casa y la familia y un $43 \%$ que el papel más importante para el hombre es ganar dinero. Parece que en lo que se refiere a estereotipos no hemos avanzado mucho en 30 años. Junto a las razones de pérdida de ingresos y la amenaza que eso supone para el papel del hombre como proveedor del sustento familiar, Hayes ahonda en los estereotipos señalando: la pérdida de estatus que experimenta un hombre cuando trabaja en campos tradicionalmente considerados femeninos, cuestionamiento de su identidad sexual debido a la homofobia, resistencia a trabajar en una posición de subordinación respecto a una mujer, discriminación de los padres sobre aquellos chicos que no exhiben una conducta acorde a su sexo o estereotipos de género en la escuela.

Pese a las definiciones más o menos consensuadas que aluden a la concentración tanto de hombres como de mujeres en diferentes campos, la segregación horizontal se ha venido entendiendo como la infrarrepresentación de la mujer en la ciencia. Pero esta es una versión parcial del problema y mientras no abordemos igualmente la infrarrepresentación de los hombres en muchas de las disciplinas académicas, seguiremos sin dar con los auténticos factores que explican la discriminación horizontal y sin medidas políticas para combatirlas.

\section{TECHO DE CRISTAL REFORZADO}

Cuando combinamos la segregación horizontal con la vertical esto es, la (im) posiblidad de las mujeres de alcanzar los puestos superiores de la jerarquía académica, encontramos lo que se ha denominado un techo de cristal reforzado. Es decir, en aquellas disciplinas masculinizadas las mujeres tienen aún más problemas para acceder a los puestos superiores de la jerarquía. Esto es 
fácilmente observable en los porcentajes de personal docente e investigador de la universidad. El porcentaje de catedráticas en la universidad española para el curso 2014-15 es de un 20,8\%. Si lo descomponemos en las cinco grandes ramas de conocimiento tenemos:

\begin{tabular}{|l|c|}
\hline \multicolumn{1}{|c|}{ Rama de conocimiento } & $\begin{array}{c}\text { Categoría } \\
\text { Catedráticas }^{12}\end{array}$ \\
\hline Ingeniería & 11.8 \\
\hline Ciencias Naturales & 21.2 \\
\hline Humanidades & 27.5 \\
\hline Ciencias sociales & 21.8 \\
\hline Ciencias de la salud & 22.8 \\
\hline Total & 20.8 \\
\hline
\end{tabular}

Tabla 2. Porcentaje de catedráticas por rama de conocimiento.

Efectivamente parece haber un techo reforzado en Ingeniería donde el porcentaje de catedráticas cae casi a la mitad respecto del total. También se cumple si miramos aquellas áreas más masculinizadas dentro de los agregados: por ejemplo, Lógica y Filosofía de la Ciencia, una de las áreas de conocimiento de Filosofía, tiene un porcentaje de catedráticas de $9.3 \%{ }^{13}$. Tenemos que ir a Ingeniería Nuclear (9.1\%) para encontrar un porcentaje tan bajo de catedráticas como hay en Lógica y Filosofía de la Ciencia. Prehistoria tiene un 11,8\%, Física tiene menos de un $7 \%$. Puede plantearse que la razón obvia es que, al haber menos mujeres en las áreas más masculinizadas, habrá menos mujeres que lleguen a los puestos altos de la jerarquía. Es decir, hay una correlación entre el número de mujeres en una rama y el número de catedráticas.

Siguiendo la misma lógica, se podría esperar que una rama tan feminizada como Ciencias de la Salud contara con un porcentaje de catedráticas muy

12. El porcentaje de catedráticas se ha obtenido de la agregación de los porcentajes de catedráticas por áreas de conocimiento lo que da porcentajes ligeramente distintos a los ofrecidos en la página del MECD.

13. En general en todas las áreas de filosofía el número de catedráticas está por debajo del $12 \%$. 
superior al promedio que es, recordemos, de 20,8\%. Pero comprobamos que no, el porcentaje es sólo dos puntos superior al promedio.

Veamos qué es lo que se ha feminizado en el ámbito de Ciencias de la Salud. En la tabla se incluyen catedráticas y titulares, así como el porcentaje del PDI por área de conocimiento.

\begin{tabular}{|l|c|c|c|}
\hline & \multicolumn{3}{|c|}{ Categoría } \\
\hline Subcampos en salud & Catedráticas & Titulares & PDI total \\
\hline Traumatología & 0 & 0 & 10.1 \\
\hline Urología & 0 & 0 & 10.4 \\
\hline Cirugía & 1.9 & 5.6 & 16.3 \\
\hline Obstetricia y ginecología & 0 & 15.4 & 37.9 \\
\hline Enfermería & 20 & 68.4 & 68.9 \\
\hline Farmacia & $43.4^{14}$ & 71.7 & 63.4 \\
\hline
\end{tabular}

Tabla 3. Porcentaje de mujeres catedráticas, titulares y de PDI total de algunos campos seleccionados dentro de la rama de conocimiento Ciencias de la Salud. ${ }^{14}$

Observamos que no hay ninguna catedrática en Traumatología, ni en Urología, hay una catedrática de un total de 52 en Cirugía (1.9\%) pero lo que es aún peor no hay tampoco ninguna catedrática en Obstetricia y Ginecología. ¿Qué pasa con el extremo opuesto de la tabla, es decir en aquellas titulaciones donde las mujeres son una mayoría abrumadora como Enfermería o Fisioterapia? Hay un 20\% de catedráticas en Enfermería (1 de 5) y ninguna en Fsioterapia. Lo más relevante es que Ciencias de la Salud, el área que se percibe como más feminizada, es la única, junto con Ingeniería, que no presenta catedráticas en algunas de sus áreas $(7 \mathrm{de} 30)^{15}$, por lo que la correlación mencionada entre el número de mujeres en una rama y el número de catedráticas, no se sostiene. No parece que las ciencias de la salud se hayan feminizado por igual. Los hombres siguen reteniendo las especialidades médicas más prestigiosas y las

14. En el cómputo se ha unido el área de farmacología $(41,2 \%)$ con el de Farmacia y tecnología farmacéutica $(51,9 \%)$.

15. Estas son: Fisioterapia, Obstetricia y Ginecología, Otorrinolaringología, Pediatría, Psiquiatría, Traumatología y Ortopedia y Urología. 
mujeres parecen quedarse en los puestos base. Pastor (et al.) en un análisis de la situación de la mujer en las profesiones sanitarias afirman:

los procesos de feminización de determinadas profesiones no implican necesariamente una mejora de las trayectorias profesionales de las mujeres en relación con su formación académica; y que los puestos de responsabilidad (también en sectores muy feminizados en su base, como el sanitario) no están distribuidos de manera proporcional entre hombres y mujeres, sino que la presencia de ellos sigue siendo mayoritaria en estos puestos. (499)

La feminización de la rama de Ciencias de la Salud que se produce desde principio de los años 90 no ha venido acompañada de una disminución de la segregación vertical. Sin embargo, la situación de Farmacia destaca por ser absolutamente anómala, con un 43 \% de catedráticas, igual que la de Biología con un $31.5 \%^{16}$. Son este tipo de cuestiones las que deben ser investigadas si queremos tener información que nos ayude a tomar decisiones políticas en otros sectores del sistema de investigación. Igual que en los casos de matrícula de estudiantes, las ramas de conocimiento ocultan en el caso del personal docente e investigador grandes disparidades.

\section{CONCLUSIONES}

a. Desde que contamos con indicadores desagregados por género, la segregación horizontal en el sistema de investigación español no ha variado, si acaso ha empeorado en Ciencias Naturales, mientras el resto de los indicadores permanece prácticamente constante.

b. Los datos agregados ocultan diferencias importantes entre distintas áreas de conocimiento que pertenecen a una misma rama. Un análisis más detallado de estos agregados muestra que algunas disciplinas tienen un peso desproporcionado en el promedio de participación de la mujer en la rama, como es el caso de Enfermería en Ciencias de la Salud, Educación en Ciencias Sociales o Biología en Ciencias, lo que distorsiona los datos de participación de las mujeres en esos campos.

16. En el caso de biología se ha unido las catedráticas de biología celular y bioquímica y biología molecular.

Feminismo/s 31, junio 2018, pp. 231-250 
c. La concentración exclusiva en las disciplinas STEM, en lo que se refiere a segregación horizontal, ha obviado el hecho de que algunas disciplinas en Humanidades presentan una segregación horizontal igual o superior a las Ingenierías. Ninguno de los factores explicativos propuestos para la segregación horizontal, fundamentalmente el peor desempeño de las mujeres en Matemáticas y su superioridad en habilidades lingüísticas, explica la situación en estos campos, lo que parece indicar, una vez más, que son otros los factores que explican la segregación horizontal.

d. En el análisis debe indagarse tanto en las elecciones femeninas como en las masculinas y no sólo deben tomarse medidas que alienten la entrada de las mujeres en Física o Ingeniería, sino la de los hombres en Educación, Medicina o Filología.

e. Si atendemos a las elecciones que hacen los estudiantes más allá de los estereotipos, comprobamos que sólo hay 1,5 puntos porcentuales de diferencia entre la proporción de chicos y chicas que eligen ciencia, pero que en ambos casos dista mucho de ser una opción preferida por ninguno de los sexos. Las medidas políticas deben alentar las vocaciones científicas eliminando los estereotipos de género asociados a la profesión.

f. Los datos muestran que la feminización de algunos campos está muy lejos de haber contribuido a mejoras en la segregación vertical. De hecho, se constata que la feminización no llega a los puestos de prestigio.

No parece que los estudios de indicadores estén incidiendo en la mejora de las cifras de las mujeres en cuestiones de discriminación horizontal. La agenda de investigación deberá incluir en un futuro el investigar la situación en nuestros grados y áreas de conocimiento y no en los agregados estadísticos de las ramas; es decir, ir más allá del enfoque basado en las disciplinas de Ciencias, Matemáticas, Ingeniería y Tecnología, y analizar especialmente aquellas disciplinas que constituyen anomalías dentro de su campo por estar muy masculinizados o muy feminizados, por ejemplo, Filosofía, Arquitectura, Ingeniería, Química o Biología. Tras quince años de medidas políticas y construcción de indicadores, es hora de poner unos y otros en relación, intentando establecer qué medidas políticas han supuesto un cambio sustancial en lo que respecta a la discriminación de las mujeres en la investigación y en qué disciplinas. 
Finalmente, debe investigarse cuáles son los factores que están detrás de la elección de carrera tanto en hombres como en mujeres.

\section{REFERENCIAS BIBLIOGRÁFICAS}

European Commission. Science policies in the European Union: Promoting excellence through mainstreaming gender equality. Luxembourg: Office for Official Publications of the European Communities, 1999.

-. She Figures 2003. Luxembourg: Office for Official Publications of the European Communities, 2004.

-. Meta-analysis of gender and science research. Luxembourg: Publications Office of the European Union, 2012.

-. Special Eurobarometer 465. Gender Equality 2017. Gender Equality, Stereotypes, and Women in Politics. Luxembourg: Directorate-General for Justice and Consumers and co-ordinated by the Directorate-General for Communication, 2017.

Gómez Rodríguez, Amparo. La estirpe maldita. La construcción científica de lo femenino. Madrid: Ediciones Minerva, 2004.

Hayes, Rader, "Men's Decisions To Enter or Avoid Nontraditional Occupations". The Career Development Quaterly 35 (1986): 89-101.

LEY ORGÁNICA 3/2007, de 22 de marzo, para la igualdad efectiva de mujeres y hombres. Boletín Oficial del Estado, Pag. 12611, 23 de marzo de 2007.

MECD. Panorama de la educación. Indicadores de la OCDE 2017. Informe español. Madrid: Dirección General de Evaluación y Cooperación Territorial Instituto Nacional de Evaluación Educativa, 2017.

NAS. Beyond Bias and Barriers: Fulfilling the Potential of Women in Academic Science and Engineering. Washington DC: National Academic Press, 2007.

OCDE. Manual on the measurement of human resources devoted to SET. "Canberra Manual”. Brussels, Luxembourg: OCDE, 1995.

OCDE. Manual de Frascati. Propuesta de Norma Práctica para Encuestas de Investigación y Desarrollo Experimental. París: FECYT, 2003.

Pastor, Inmaculada, Ángel Belzunegui, Paloma Pontón. "Mujeres en sanidad: entre la igualdad y la desigualdad". Cuadernos de Relaciones Laborales 30 (2012): 497-518.

Pérez Sedeño, Eulalia, Marta I. González, María Jesús Santesmases, Arantxa Martín, Paloma de Villota, Ana Guill, Esther Ortega, Verónica Sanz y Adriana Kiczkowsk. La situación de las mujeres en el sistema Educativo de ciencia y tecnología en 
España y su contexto internacional. Programa de análisis y estudios de acciones destinadas a la mejora de la Calidad de la enseñanza superior y de actividades del profesorado Universitario (REF: S2/EA2003-0031)2003. http://www. ohchr.org/Documents/Issues/CulturalRights/ConsultationEnjoyBenefits/ UNESCOLASITUACIANDELASMUJERESENELSISTEMA.pdf

RD 415/2015, Boletín Oficial del Estado, p. 50319, 17 de junio de 2015.

Rubio, Isabel. "Hay niñas que sí sueñan con ser como Marie Curie". El País 22 noviembre 2017. Edición digital. Consultado el 05/12/2017.

Torres, Obdulia. "Science and Gender Indicators: a critical review". International Journal of Gender, Science and Techonoloy 4 (2012): 24-47.

UNESCO. Clasificación Internacional Normalizada de la Educación CINE 2011. Montreal, Quebec: Instituto de Estadística de la UNESCO, 2013. 